\title{
Cochrane eyes and vision
}

\author{
Sophie Z. Gu${ }^{1} \cdot$ David S. Friedman ${ }^{1} \cdot$ Augusto Azuara-Blanco ${ }^{2}$
}

Received: 7 December 2018 / Accepted: 7 December 2018 / Published online: 19 February 2019

(c) The Royal College of Ophthalmologists 2019

Practicing evidence-based medicine is easier said than done. Few would question the value of using current best evidence in making critical healthcare decisions. The problem, however, is that providers and patients alike are frequently inundated with vast amounts of information derived from multiple (and often contradictory) sources. Information overload makes it challenging to fully synthesize research findings.

Systematic reviews attempt to address this difficulty-they critically assess the evidence on a given topic. One of the most widely used sources of systematic reviews is the Cochrane Database of Systematic Reviews (CDSR). Since its inception in 1996, the CDSR has published numerous systematic reviews of healthcare interventions, ranging from pharmacological and surgical treatments to preventive measures and diagnostic tests. This includes those pertaining to eye disease and visual impairment, produced by a division called Cochrane Eyes and Vision (CEV, https:// eyes.cochrane.org/).

The methodology behind each Cochrane review is rigorous and transparent (i.e., follows a published protocol). After a team of authors identifies all prior primary research studies on the topic in question, a strict vetting process ensues. Each study is judged not only on the presence of conclusive evidence, but also, importantly, on quality of data and risk of bias. When feasible, meta-analysis is done to integrate all available data, increasing statistical power and precision. The results are summarized in a table and, if possible, a forest plot to provide a reliable and user-friendly synthesis of the evidence on the given topic. To ensure accessibility to the general public, each Cochrane review also includes a plain language summary.

Sophie Z. Gu

Zgu8@jhmi.edu

1 John Hopkins University, Baltimore, MD, USA

2 Queen's University, Belfast, UK
By evaluating large bodies of evidence in a structured and transparent manner, Cochrane reviews provide insights that are not captured in a single study. Moreover, the reviews are living documents-as additional evidence emerges, reviews are periodically updated. This allows providers, patients, researchers, and policy makers to understand the latest developments, keeping pace with the rapidly changing landscape of medicine.

An early example of the impact of Cochrane reviews on health outcomes is illustrated in the logo.

\section{() Cochrane}

The forest plot within the logo represents the summary results of a study on the effectiveness of oral corticosteroids administered to pregnant women prior to preterm delivery [1]. Although several clinical trials had previously been conducted, it was not until the publication of Cochrane's systematic review that this intervention became widely used, saving thousands of premature babies.

The CEV Group has also conducted impactful reviews of novel drug therapies for eye diseases. One excellent example is Cochrane's review of the use of anti-vascular endothelial growth factor (anti-VEGF) for neovascular age-related macular degeneration (AMD) [2]. Anti-VEGF agents are injected intravitreally and aim to prevent the growth of abnormal blood vessels in the back of the eye. Examining 12 randomized controlled trials involving a total of 5496 patients across five continents, Cochrane compared the three commercially available drugs at the time of the review (pegaptanib, ranibizumab, and bevacizumab). All three were found to have protective effects in neovascular AMD, but the latter two yielded larger improvements in visual acuity than pegaptanib. Furthermore, the authors noted that while there was little difference in therapeutic outcome between bevacizumab and ranibizumab, ranibizumab was considerably more costly. 
Cochrane has also synthesized evidence on the efficacy of visual rehabilitation, the effects of different models of eye care, and the performance and accuracy of diagnostic tests for eye diseases. Besides influencing healthcare decision-making, such reviews often expose important gaps in knowledge, encouraging the conduct of primary research and informing future research directions.

Not all reviews are created equal. Regarding the increasing number of poor-quality systematic reviews that have been published in the literature, John Ionannadis, a highly cited researcher and expert on research methodology, has said, “... the large majority of produced systematic reviews and metaanalyses are unnecessary, misleading, and/or conflicted" [3]. The validity, trustworthiness, and relevance of Cochrane reviews are thus more important now than ever.

Beginning in February, Eye will feature Cochrane Eye and Vision reviews. Although Cochrane reviews were originally conceived as electronic publications, highlighting reviews in this journal will ensure wider readership. With enhanced distribution of Cochrane's critical insights into healthcare interventions, providers will be able to better implement evidence-based medicine and improve patient care.

\section{Compliance with ethical standards}

Conflict of interest The authors declare that they have no conflict of interest.

Publisher's note: Springer Nature remains neutral with regard to jurisdictional claims in published maps and institutional affiliations.

\section{References}

1. Crowley P. Prophylactic corticosteroids for preterm birth. Cochrane Database Syst Rev. 2000;CD000065.

2. Solomon SD, Lindsley K, Vedula SS, Krzystolik MG, Hawkins BS. Anti-vascular endothelial growth factor for neovascular agerelated macular degeneration. Cochrane Database Syst Rev. 2014; CD005139.

3. Ioannidis JP. The mass production of redundant, misleading, and conflicted systematic reviews and meta-analyses. Milbank Q. 2016;94:485-514. 